\title{
Asymmetrical Impact of Allee Effect on a Discrete-Time Predator-Prey System
}

\author{
Wenting Wang, Yujuan Jiao, and Xiuping Chen \\ School of Mathematics and Computer Science, Northwest University for Nationalities, \\ Lanzhou 730030, China \\ Correspondence should be addressed to Wenting Wang; sci-c-author@hotmail.com
}

Received 28 August 2012; Revised 2 March 2013; Accepted 8 March 2013

Academic Editor: Vu Phat

Copyright (C) 2013 Wenting Wang et al. This is an open access article distributed under the Creative Commons Attribution License, which permits unrestricted use, distribution, and reproduction in any medium, provided the original work is properly cited.

A discrete-time predator-prey model is proposed with Leslie-type numerical response, and the asymmetrical influence of Allee effect on the proposed system is investigated. By mathematical analysis, locally stable conditions for the equilibrium points of the considered systems with or without Allee effect are obtained firstly. Furthermore, numerical simulation is used to verify the results and detect some new outcomes. The results show that Allee effect on predator leads the system to its stable state in much longer time. Conversely, the prey population with Allee effect makes it much faster. In particular, a large value of Allee effect on prey results in periodic dynamics of the system.

\section{Introduction}

In recent years, the Allee effect has drawn a considerable attention in almost every aspect of ecology and conservation. This effect, a reduction of individual fitness at low population density, may come about through a number of mechanisms, such as mate-search difficulty, increased predation risk due to failing flocking or schooling behavior, or reduced foraging efficiency in social predators [1-4]. Strong Allee effects lead to threshold population densities, below which the population growth is negative and the population is likely to go extinct $[5,6]$. Such effects may be observed on different organisms including vertebrates, invertebrates, and plants. The effect usually saturates or vanishes as the population size gets larger. Although some work has shown that Allee effects might play a stabilizing and protective role [7-10], most studies have highlighted its negative impact on population persistence $[4,5,11-14]$.

Many investigators have explored the consequences and the different ways of incorporating Allee effects into deterministic continuous-time population models [15-19]. For example, several recent papers have explored the role that Allee effects played in competition and predator-prey interactions. Wang et al. [15] highlighted that introducing an Allee effect into a Lotka-Volterra competitor system would destabilize it. Courchamp et al. [16] have shown that cooperative species, because they are sensitive to an Allee effect, are more sensitive to interactions with natural enemies such as competitors or predators. Some studies have also shown that structured prey populations and stage-specific predation lead to Allee effects in the (top) predator population [17-19].

In this paper, we are interested in deterministic discretetime population models incorporating Allee effects. Considering many researches and studies in mathematical biology, we can say that discrete-time models described by difference equations are more appropriate and realistic than the continuous-time models in the case of populations having distinct, nonoverlapping generations. In particular, discretetime models can also provide more efficient computational models for numerical simulations and richer dynamics can be observed from them. In two previous papers [20, 21], the stability of a simple discrete-time predator-prey system with Holling's type I functional response is studied, while only the prey obeys the Allee effect. However, Allee effect can occur not only on prey but also on predator populations in real ecosystems. If the predator and prey have the Allee effect respectively, what will happen? For these issues, we propose 
a discrete-time Leslie-type predator-prey model and explore the asymmetrical impact of Allee effect on that system.

This paper is organized as follows. In Section 2, we briefly introduce the mathematical model which we will analyze and obtain the local stability conditions for the equilibrium points. After that, in Section 3, we study local behavior of the equilibrium points when the predator and prey population are subject to an Allee effect, respectively. In Section 4, some numerical simulations are given and the asymmetrical impact of Allee effect on the system is investigated. Finally, the last section of the paper is devoted to the discussion and remarks.

\section{The Mathematical Model and Stability Analysis}

We introduce the following discrete-time predator-prey system:

$$
\begin{gathered}
x_{n+1}=x_{n}+r x_{n}\left(1-\frac{x_{n}}{K}\right)-\alpha x_{n} y_{n}, \\
y_{n+1}=y_{n}+\beta y_{n}\left(1-\frac{y_{n}}{x_{n}}\right),
\end{gathered}
$$

where $r, \alpha$, and $\beta$ are positive constants, and $K$ is the carrying capacity. The parameter $r$ is the maximal growth rate of the prey, $\alpha$ is the predation parameter, and $\beta$ is the coefficient of food utilization. The variables $x_{n}, y_{n}$ represent the populations density of prey and its predator at the $n$th generation, respectively. Here, the term $x_{n}+r x_{n}\left(1-x_{n} / K\right)$ stands for the increasing rate of the prey population in the absence of predator, while the term $\alpha x_{n} y_{n}$ represents the decreasing rate due to predation. Finally, the term $y_{n}+$ $\beta y_{n}\left(1-y_{n} / x_{n}\right)$ stands for the variation of predator density which depends on both the number of preys and predators. The predator population grows logistically with a carrying capacity, $x_{n}$, that is proportional to the number of prey. This was firstly introduced by Leslie [22]. Notice that, if the predator density disappears in this model, then the prey density satisfies the discrete logistic-type model.

Next we will consider the local stability of the equilibrium points of system (1). To get this, firstly observe that corresponding equilibrium points of system $(1)$ are $(K, 0)$ and $\left(x_{0}^{*}, y_{0}^{*}\right)$, where

$$
x_{0}^{*}=y_{0}^{*}=\frac{r K}{r+\alpha K} \text {. }
$$

Clearly, $\left(x_{0}^{*}, y_{0}^{*}\right)$ is the unique positive equilibrium point of system (1).

The Jacobian matrix of system (1) at the equilibrium point $(K, 0)$ is

$$
J_{0}^{1}=\left(\begin{array}{cc}
1-r & -\alpha \\
0 & 1+\beta
\end{array}\right)
$$

Hence, $(K, 0)$ is not asymptotically stable. Usually, such a point is called a nonhyperbolic equilibrium point.
For the positive equilibrium point $\left(x_{0}^{*}, y_{0}^{*}\right)$, the Jacobian matrix is given by

$$
J_{0}^{2}=\left(\begin{array}{cc}
1-\frac{r^{2}}{r+\alpha K} & -\frac{\alpha r K}{r+\alpha K} \\
\beta & 1-\beta
\end{array}\right) .
$$

Then we have the corresponding characteristic equation to the matrix $J_{0}^{2}$ as follows:

$$
\Phi_{0}(\lambda)=\lambda^{2}-\left(2-\beta-\frac{r^{2}}{r+\alpha K}\right) \lambda+1-\beta-\frac{r^{2}}{r+\alpha K}+\beta r .
$$

It follows from the well-known Jury conditions (see in [23]) that the modulus of all roots of (5) is less than 1 if and only if

$$
\Phi_{0}(1)>0, \quad \Phi_{0}(-1)>0, \quad \operatorname{det} J_{0}^{2}<1 .
$$

Clearly, $\Phi_{0}(1)=\beta r>0$ for any $\beta, r>0$. On the other hand, $\Phi_{0}(-1)>0$ holds if and only if $2\left(2-\beta-r^{2} /(r+\alpha K)\right)+$ $\beta r>0$, which implies

$$
\frac{r}{2}-\frac{r^{2}}{\beta(r+\alpha K)}>1-\frac{2}{\beta}
$$

Again, the final inequality $\operatorname{det} J_{0}^{2}<1$ holds if and only if $1-\beta-r^{2} /(r+\alpha K)+\beta r<1$, which is equivalent to

$$
r-\frac{r^{2}}{\beta(r+\alpha K)}<1 .
$$

Now combining (7) and (8), we can get the following conclusion.

Theorem 1. The positive equilibrium point $\left(x_{0}^{*}, y_{0}^{*}\right)$ of system (1) is locally asymptotically stable if

$$
1+\frac{r}{2}-\frac{2}{\beta}<r-\frac{r^{2}}{\beta(r+\alpha K)}<1 .
$$

The following result is an immediate consequence of Theorem 1.

Corollary 2. The positive equilibrium point $\left(x_{0}^{*}, y_{0}^{*}\right)$ of system (1) is unstable if and only if

$$
1-\frac{2}{\beta}>\frac{r}{2}-\frac{r^{2}}{\beta(r+\alpha K)} \quad \text { or } \quad r-\frac{r^{2}}{\beta(r+\alpha K)}>1 .
$$

\section{Asymmetrical Allee Effect}

3.1. Allee Effect on Predator Population. Many predators may be easier to experience an Allee effect because a low number of viable hunters will suffer ineffectiveness in predation [24]. In order to show the influence of Allee effect on the dynamics of system (1), we consider the system as subjecting to an Allee effect on predator population and obtain the following model:

$$
\begin{aligned}
& x_{n+1}=x_{n}+r x_{n}\left(1-\frac{x_{n}}{K}\right)-\alpha x_{n} y_{n}, \\
& y_{n+1}=y_{n}+\beta y_{n}\left(\frac{y_{n}}{y_{n}+u_{1}}-\frac{y_{n}}{x_{n}}\right),
\end{aligned}
$$


where $y_{n} /\left(y_{n}+u_{1}\right)$ denotes the predator with the "weak" Allee effect, and $u_{1}$ the Allee constant satisfying the assumption

$$
0<u_{1}<K
$$

All the equilibrium points of system $(11)$ are $(K, 0)$ and $\left(x_{u_{1}}^{*}, y_{u_{1}}^{*}\right)$, where

$$
x_{u_{1}}^{*}=\frac{K\left(r+\alpha u_{1}\right)}{r+\alpha K}, \quad y_{u_{1}}^{*}=\frac{r\left(K-u_{1}\right)}{r+\alpha K} .
$$

Under the above assumption, the predator-prey system (11) has unique positive equilibrium point $\left(x_{u_{1}}^{*}, y_{u_{1}}^{*}\right)$. It is clear that $x_{u_{1}}^{*}>x_{0}^{*}$ and $y_{u_{1}}^{*}<y_{0}^{*}$.

We can easily see that $(K, 0)$ is also a nonhyperbolic equilibrium point of system (11). Next, we will focus on the positive equilibrium point $\left(x_{u_{1}}^{*}, y_{u_{1}}^{*}\right)$. The Jacobian matrix is

$$
J_{u_{1}}=\left(\begin{array}{cc}
1-\frac{r}{K} x_{u_{1}}^{*} & -\alpha x_{u_{1}}^{*} \\
\beta\left(1-\frac{u_{1}}{x_{u_{1}}^{*}}\right)^{2} & 1-\beta\left(1-\frac{u_{1}}{x_{u_{1}}^{*}}\right)^{2}
\end{array}\right) .
$$

Thus the matrix $J_{u_{1}}$ yields the characteristic equation

$$
\Phi_{u_{1}}(\lambda)=\lambda^{2}-\left(\operatorname{tr} J_{u_{1}}\right) \lambda+\operatorname{det} J_{u_{1}}=0
$$

where

$$
\begin{aligned}
\operatorname{tr} J_{u_{1}}= & 2-\frac{r}{K} x_{u_{1}}^{*}-\beta\left(1-\frac{u_{1}}{x_{u_{1}}^{*}}\right)^{2}, \\
\operatorname{det} J_{u_{1}}= & 1-\frac{r}{K} x_{u_{1}}^{*}-\beta\left(1-\frac{u_{1}}{x_{u_{1}}^{*}}\right)^{2} \\
& +\frac{\beta(r+\alpha K)}{K} x_{u_{1}}^{*}\left(1-\frac{u_{1}}{x_{u_{1}}^{*}}\right)^{2} .
\end{aligned}
$$

According to the Jury conditions we obtain that the modulus of all roots of (15) is less than 1 which implies the equilibrium point $\left(x_{u_{1}}^{*}, y_{u_{1}}^{*}\right)$ is asymptotically stable if $\Phi_{u_{1}}(1)>0, \Phi_{u_{1}}(-1)>0$, and $\operatorname{det} J_{u_{1}}<1$.

Firstly, $\Phi_{u_{1}}(1)>0$ holds for any positive parament of system (11), since

$$
\Phi_{u_{1}}(1)=\frac{\beta(r+\alpha K)}{K} x_{u_{1}}^{*}\left(1-\frac{u_{1}}{x_{u_{1}}^{*}}\right)^{2} .
$$

Again, under the assumption $0<u_{1}<K, \Phi_{u_{1}}(-1)>0$ holds if

$$
\begin{aligned}
2[2- & \left.\frac{r}{K} x_{u_{1}}^{*}-\beta\left(1-\frac{u_{1}}{x_{u_{1}}^{*}}\right)^{2}\right] \\
& +\frac{\beta(r+\alpha K)}{K} x_{u_{1}}^{*}\left(1-\frac{u_{1}}{x_{u_{1}}^{*}}\right)^{2}>0,
\end{aligned}
$$

that is

$$
1-\frac{2}{r+\alpha u_{1}}>\left[\frac{r\left(r+\alpha u_{1}\right)}{r+\alpha K}-2\right] \frac{2 K^{2}\left(r+\alpha u_{1}\right)}{\beta r^{2}\left(K-u_{1}\right)^{2}} .
$$

Finally, det $J_{u_{1}}<1$ holds if

$$
1-\frac{r}{K} x_{u_{1}}^{*}-\beta\left(1-\frac{u_{1}}{x_{u_{1}}^{*}}\right)^{2}+\frac{\beta(r+\alpha K)}{K} x_{u_{1}}^{*}\left(1-\frac{u_{1}}{x_{u_{1}}^{*}}\right)^{2}<1,
$$

that is

$$
1-\frac{1}{r+\alpha u_{1}}<\frac{K^{2}\left(r+\alpha u_{1}\right)^{2}}{\beta r(r+\alpha K)\left(K-u_{1}\right)^{2}} .
$$

Now we obtain the following conclusion.

Theorem 3. Assuming that $0<u_{1}<K$, the positive equilibrium point $\left(x_{u_{1}}^{*}, y_{u_{1}}^{*}\right)$ of system (11) is locally asymptotically stable if the conditions (19) and (21) are satisfied.

The next result is an immediate consequence of Theorem 3.

Corollary 4. The positive equilibrium point $\left(x_{u_{1}}^{*}, y_{u_{1}}^{*}\right)$ of system (11) is unstable if and only if

$$
1-\frac{2}{r+\alpha u_{1}}<\left[\frac{r\left(r+\alpha u_{1}\right)}{r+\alpha K}-2\right] \frac{2 K^{2}\left(r+\alpha u_{1}\right)}{\beta r^{2}\left(K-u_{1}\right)^{2}}
$$

or

$$
1-\frac{1}{r+\alpha u_{1}}>\frac{K^{2}\left(r+\alpha u_{1}\right)^{2}}{\beta r(r+\alpha K)\left(K-u_{1}\right)^{2}} .
$$

When the predator is with Allee effect, the predator will decrease and the prey will increase. According to Theorem 3 and Corollary 4, the predator must improve rates of their predation and food utilization to avoid extinction (see Figure 1(a)). The predator and prey would coexist when all parameters of the system satisfy Theorem 3.

3.2. Allee Effect on Prey Population. Because of difficulties in finding mates, social dysfunctions, inbreeding depression, and dispersal cost, the prey is usually suffered from Allee effect $[5,25]$. In this section, we will consider the predatorprey system (11) as subject to an Allee effect on prey population and analyze the following system:

$$
\begin{gathered}
x_{n+1}=x_{n}+r x_{n}\left(1-\frac{x_{n}}{K}\right) \frac{x_{n}}{x_{n}+u_{2}}-\alpha x_{n} y_{n}, \\
y_{n+1}=y_{n}+\beta y_{n}\left(1-\frac{y_{n}}{x_{n}}\right),
\end{gathered}
$$

where we take $x_{n} /\left(x_{n}+u_{2}\right)$ as the Allee effect function and $u_{2}$ as the Allee constant satisfying the assumption

$$
0<u_{2}<\frac{r}{\alpha}
$$




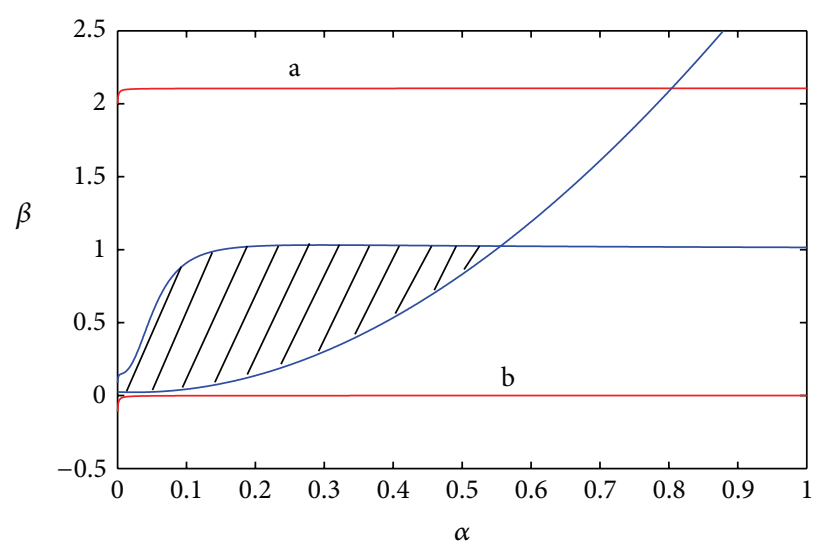

(a)

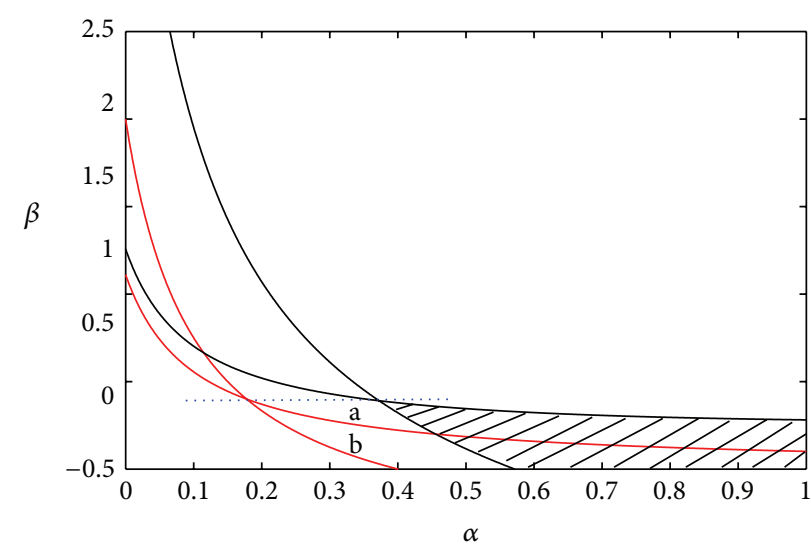

(b)

FIGURE 1: Compare the parameter regions of coexistence when the predator and prey are with Allee effect (shaded area) with those when they are without Allee effect, respectively. The area between lines $\mathrm{a}$ and $\mathrm{b}$ is the regions of coexistence when the predator-prey system is without Allee effect. (a) The situation of the predator with Allee effect. Parameter values are $r=10, K=100$, and $u_{1}=6$; (b) the situation of the prey with Allee effect. Parameter values are $r=0.1, K=100$, and $u_{2}=6$.

Then we have two equilibrium points of system (24) as $(K, 0)$ and $\left(x_{u_{2}}^{*}, y_{u_{2}}^{*}\right)$, where

$$
x_{u_{2}}^{*}=y_{u_{2}}^{*}=\frac{K\left(r-\alpha u_{2}\right)}{r+\alpha K} .
$$

Clearly, the equilibrium point $(K, 0)$ is a nonhyperbolic equilibrium point of system (24).

Under the assumption (25), the predator-prey system (24) has unique positive equilibrium point $\left(x_{u_{2}}^{*}, y_{u_{2}}^{*}\right)$. It is clear that $x_{u_{2}}^{*}<x_{0}^{*}$ and $y_{u_{2}}^{*}<y_{0}^{*}$. After some simple calculations, the Jacobian matrix of (24) turns out to be

$$
J_{u_{2}}=\left(\begin{array}{cc}
1-\omega x_{u_{2}}^{*} & -\alpha x_{u_{2}}^{*} \\
\beta & 1-\beta
\end{array}\right) \text {, }
$$

where

$$
\omega=\frac{r-\alpha u_{2}}{u_{2}+K}-\frac{\alpha u_{2}(r+\alpha K)}{r\left(u_{2}+K\right)} .
$$

Then the characteristic equation of matrix $J_{u_{2}}$ is

$$
\Phi_{u_{2}}(\lambda)=\lambda^{2}-\left(\operatorname{tr} J_{u_{2}}\right) \lambda+\operatorname{det} J_{u_{2}}=0
$$

where

$$
\begin{gathered}
\operatorname{tr} J_{u_{2}}=2-\beta-\omega x_{u_{2}}^{*}, \\
\operatorname{det} J_{u_{2}}=1-\beta-\omega x_{u_{2}}^{*}+\beta(\alpha+\omega) x_{u_{2}}^{*} .
\end{gathered}
$$

Again, by using the Jury conditions we obtain that the equilibrium point $\left(x_{u_{2}}^{*}, y_{u_{2}}^{*}\right)$ is asymptotically stable if and only if $\Phi_{u_{2}}(1)>0, \Phi_{u_{2}}(-1)>0$ and $\operatorname{det} J_{u_{2}}<1$.

We first obtain that $\Phi_{u_{1}}>0$ if and only if $\beta(\alpha+\omega) x_{u_{2}}^{*}>0$, and this implies $\alpha+\omega>0$. Now assume the following function:

$$
\varphi\left(u_{2}\right)=\alpha+\omega=\frac{(r+\alpha K)\left(r-\alpha u_{2}\right)}{r\left(K+u_{2}\right)}, \quad u_{2} \in\left[0, \frac{r}{\alpha}\right] .
$$

Since

$$
\varphi^{\prime}\left(u_{2}\right)=-\frac{(r+\alpha K)^{2}}{r\left(K+u_{2}\right)^{2}}<0,
$$

$\varphi$ is a strictly decreasing function on $[0, r / \alpha]$. Thus, $\varphi$ attains its minimum value at $u_{2}=r / \alpha$. Since $\varphi(r / \alpha)=0$, we conclude that

$$
\alpha+\omega>0 \quad \forall u_{2} \in\left(0, \frac{r}{\alpha}\right) .
$$

So $\Phi_{u_{2}}(1)>0$ if and only if the condition (25) is satisfied. Again,

$$
\begin{aligned}
& \Phi_{u_{2}}(-1)>0 \Longleftrightarrow 2\left(2-\beta-\omega x_{u_{2}}^{*}\right)+\beta(\alpha+\omega) x_{u_{2}}^{*}>0 \\
& \Longleftrightarrow \frac{\alpha K\left(r-\alpha u_{2}\right)}{\beta(r+\alpha K)}+\left(\frac{1}{\beta}-\frac{1}{2}\right) \\
& \times \frac{K\left(r-\alpha u_{2}\right)^{2}}{r\left(K+u_{2}\right)}>1-\frac{2}{\beta}, \\
& \operatorname{det} J_{u_{2}}<1 \Longleftrightarrow 1-\beta-\omega x_{u_{2}}^{*}+\beta(\alpha+\omega) x_{u_{2}}^{*} \Longleftrightarrow \frac{\alpha K\left(r-\alpha u_{2}\right)}{\beta(r+\alpha K)}+\left(\frac{1}{\beta}-1\right) \frac{K\left(r-\alpha u_{2}\right)^{2}}{r\left(K+u_{2}\right)}<1 .
\end{aligned}
$$

Now considering (34), we can get the following results.

Theorem 5. Assuming that $0<u_{2}<r / \alpha$, the positive equilibrium point $\left(x_{u_{2}}^{*}, y_{u_{2}}^{*}\right)$ of system (24) is locally asymptotically stable if

$$
\begin{aligned}
1-\frac{2}{\beta} & +\frac{K\left(r-\alpha u_{2}\right)^{2}}{2 r\left(K+u_{2}\right)} \\
& <\frac{\alpha K\left(r-\alpha u_{2}\right)}{\beta(r+\alpha K)}+\left(\frac{1}{\beta}-1\right) \frac{K\left(r-\alpha u_{2}\right)^{2}}{r\left(K+u_{2}\right)}<1 .
\end{aligned}
$$




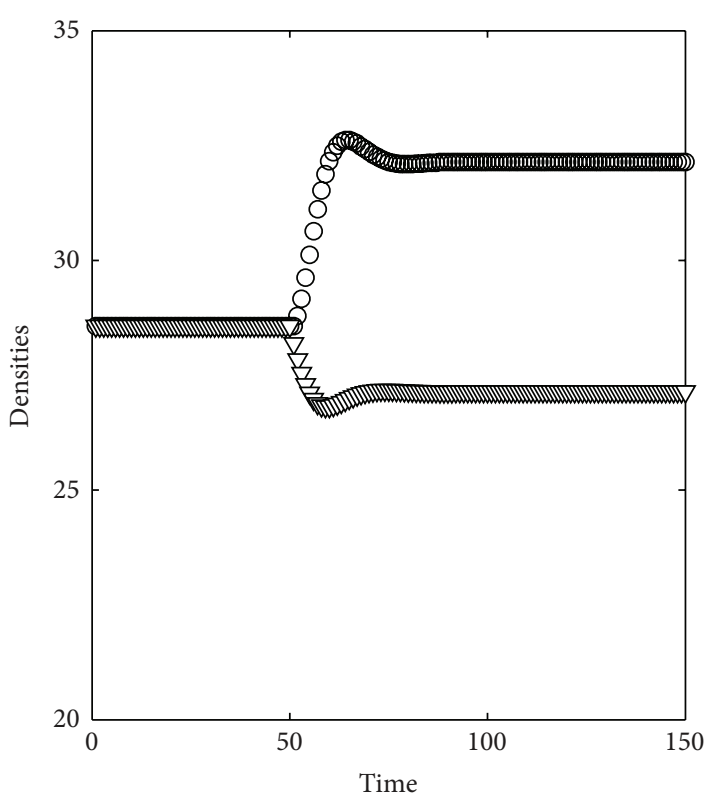

(a)

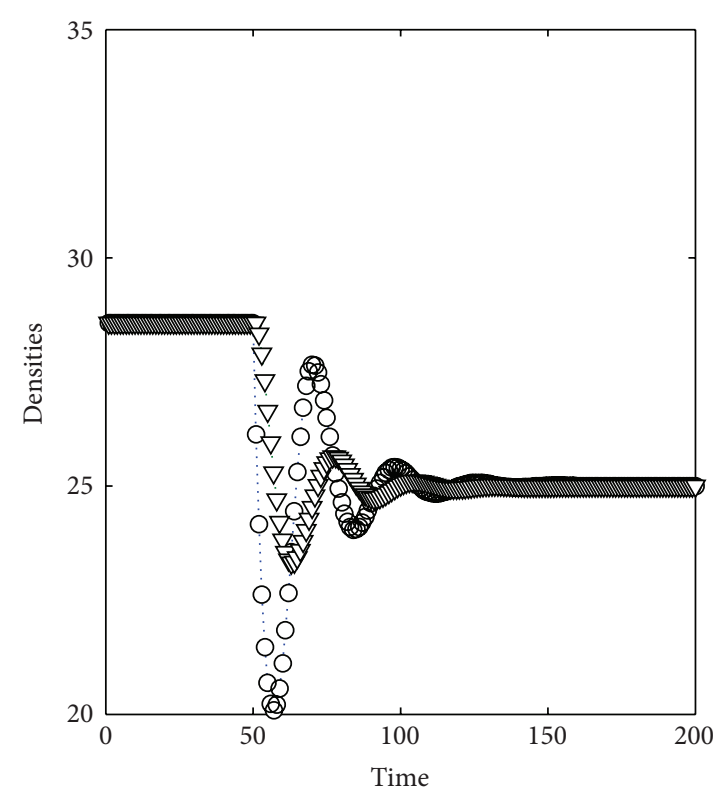

(b)

Figure 2: Typical examples of predator $(\nabla)$ and prey $(\circ)$ population dynamics. Allee effect of predator (or prey) starts to occur at $t=50$. (a) Predator with Allee effect, and (b) prey with Allee effect. Before the Allee effect $(t<50)$, the system stays in steady state. Parameter values: $r=0.8, \alpha=0.02, \beta=0.09, K=100$, and $u_{1}=u_{2}=5$.

The next result is an immediate consequence of Theorem 5 .

Corollary 6. The positive equilibrium point $\left(x_{u_{2}}^{*}, y_{u_{2}}^{*}\right)$ of system (11) is unstable if and only if

$$
1-\frac{2}{\beta}>\frac{\alpha K\left(r-\alpha u_{2}\right)}{\beta(r+\alpha K)}+\left(\frac{1}{\beta}-\frac{1}{2}\right) \frac{K\left(r-\alpha u_{2}\right)^{2}}{r\left(K+u_{2}\right)}
$$

or

$$
\frac{\alpha K\left(r-\alpha u_{2}\right)}{\beta(r+\alpha K)}+\left(\frac{1}{\beta}-1\right) \frac{K\left(r-\alpha u_{2}\right)^{2}}{r\left(K+u_{2}\right)}>1 .
$$

When the prey is with Allee effect, both densities of prey and predator will decrease. According to Theorem 5 and Corollary 6 , decrease of the rates of prey predation and food utilization is the way of the predator and prey to keep coexistence (see Figure 1(b)).

\section{Numerical Simulations and Analysis}

In this section, some numerical simulations are performed to verify our theoretical results showed in the previous sections and further analyze the asymmetrical influence of Allee effect on the predator-prey system. We used the MATLAB for these computations. Mainly, we show the asymmetrical impact of Allee effect on the predator-prey system in the following three ways: (i) both species without Allee effect (model (1)), (ii) only predator with Allee effect (model (11)), and (iii) only prey with Allee effect (model (24)).
We assume that $K=100$ throughout this section.

Typical population dynamics are shown in Figure 2, where (a) and (b) denote the cases that the predator species and prey start to be with Allee effect at $t=50$, respectively. Before the Allee effect, the system stays in steady state. Figure 2 exhibits the asymmetrical influence of Allee effect between prey and predator. It is found from Figure 2(a) that the density of prey species increases and predator decreases in the final equilibrium by the Allee effect in predator (model (11), $u_{1}=5$ ). In contrast, when the prey species obeys the Allee effect (model (24), $u_{2}=5$ ), then both species finally decrease their population size (see Figure 2(b)).

In Figure 3, we illustrate the trajectories of predator and prey densities in systems (1), (11), and (24). Here (a1) and (b1) show the trajectories of predator and prey densities in models (1), (a2), and (b2) correspond to model (24) that the predator is subject to the Allee effect; however, (a3) and (b3) correspond to model (11) that the prey is subject to the Allee effect. We use $r=0.7, \alpha=0.05, \beta=0.6$, $u_{1}=u_{2}=5$, and the initial conditions $x_{0}=25, y_{0}=$ 15 in Figure 3(a1-a3). We see from Figure 3(a1-a3), that, when the predator population is subject to an Allee effect, the local stability of the equilibrium point decreases and it takes a long time to approximate to the corresponding equilibrium point, while the prey population obeys an Allee effect, the local stability of the equilibrium point increases and trajectory of the solution approximates to the corresponding equilibrium point much faster. Furthermore, Figure 3(b1-b3) presents peculiar trajectories of predator and prey population by taking $r=3, \alpha=0.04, \beta=0.04, u_{1}=u_{2}=8$, and the initial conditions $x_{0}=30, y_{0}=20$. we find 


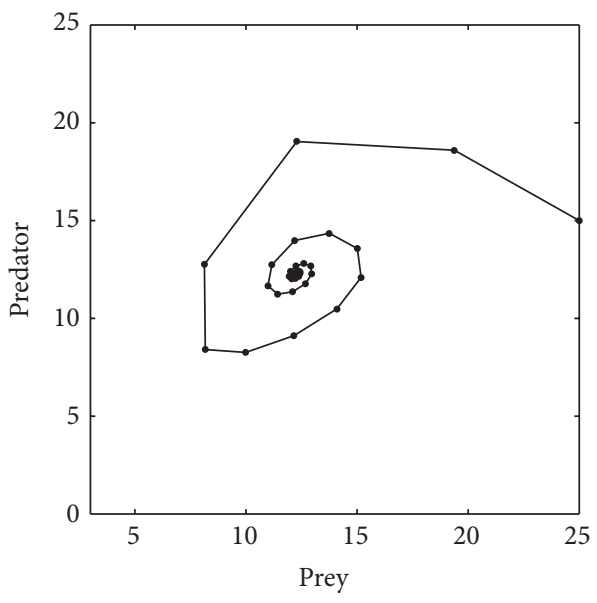

(a1)

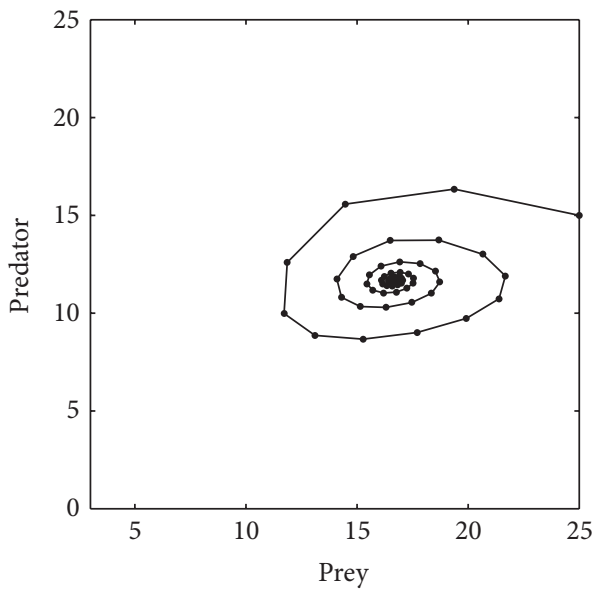

(a2)

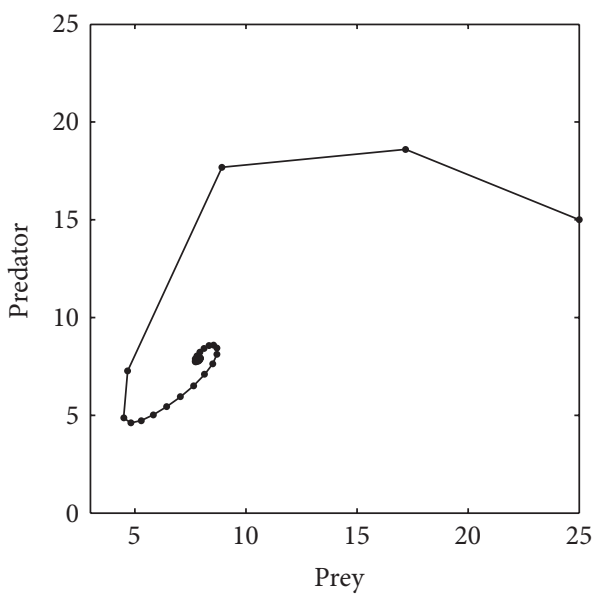

(a3)

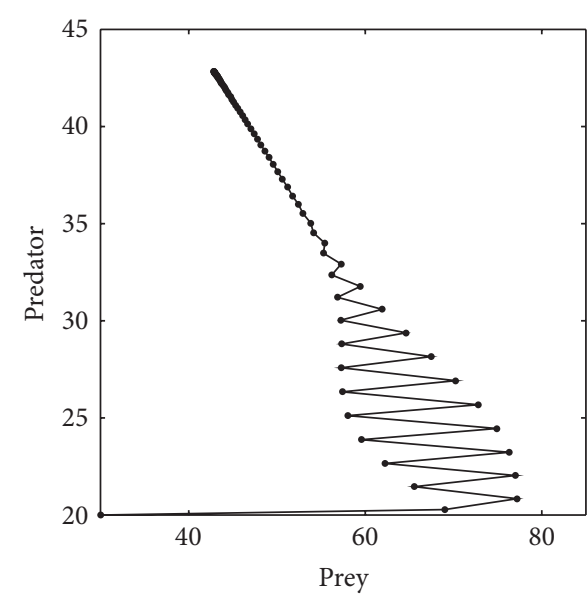

(b1)

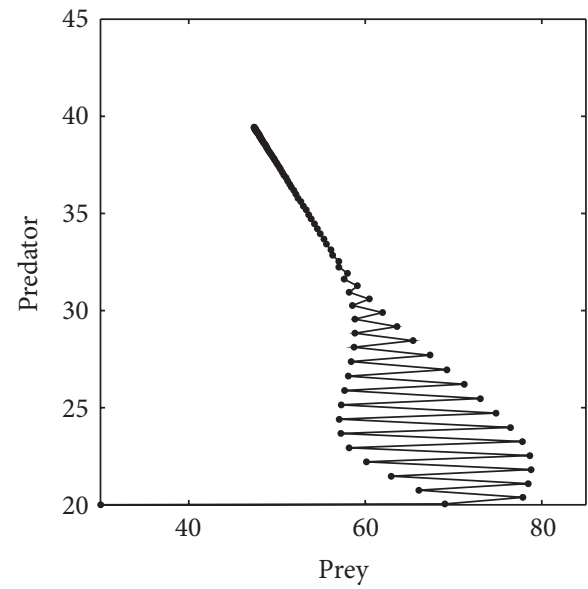

(b2)

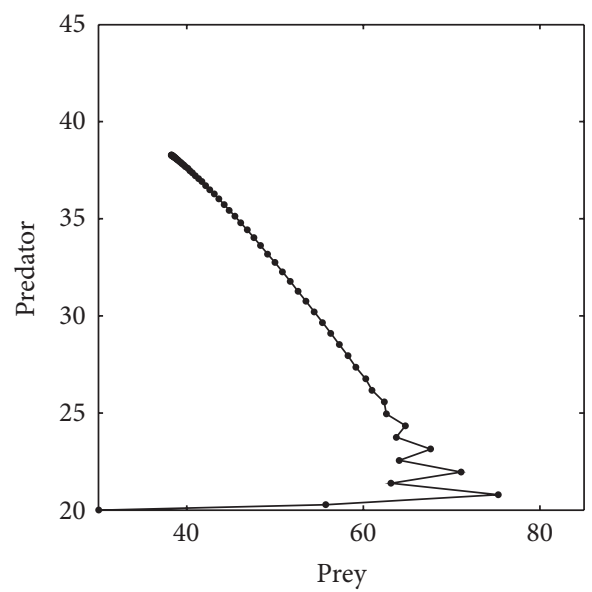

(b3)

FIGURE 3: The trajectories of the prey and predator population described by model (1) ((a1) and (b1)), model (11) ((a2) and (b2)), and model (24) ((a3) and (b3)). Parameter values: (al-a3): $r=0.7, \alpha=0.05, \beta=0.6, K=100 ; u_{1}=u_{2}=5, x_{0}=25$, and $y_{0}=15$; (b1-b3): $r=3$, $\alpha=0.04, \beta=0.04, K=100, u_{1}=u_{2}=8, x_{0}=30$, and $y_{0}=20$. 


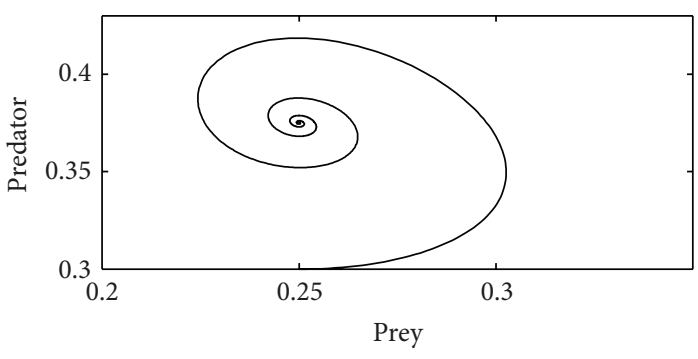

(a1)

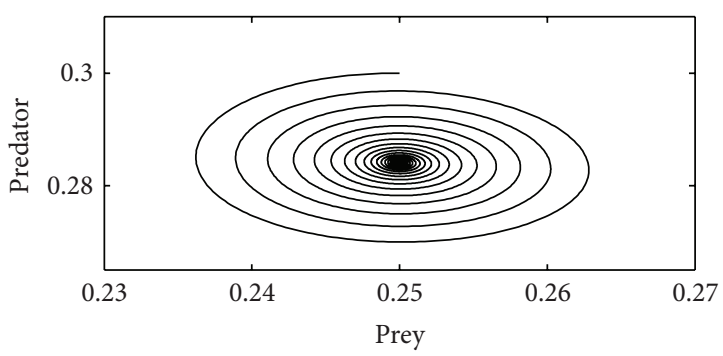

(a2)

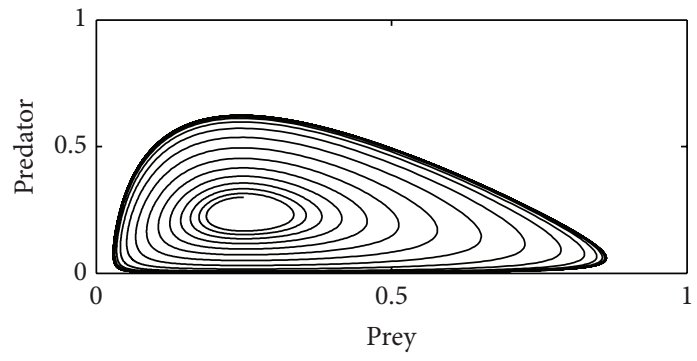

(a3)

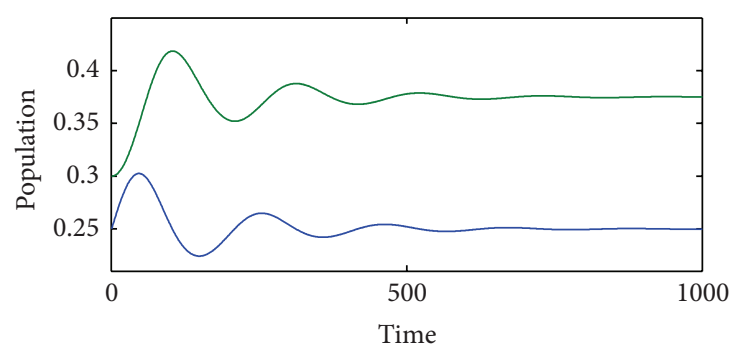

(b1)

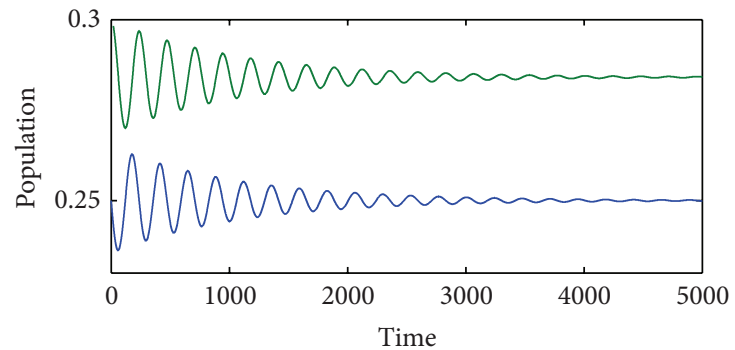

(b2)

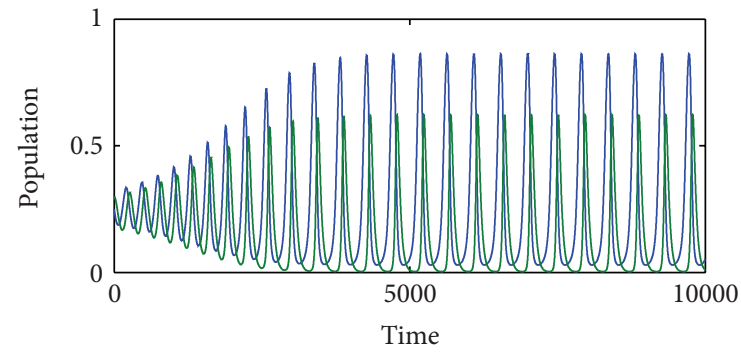

(b3)

FIgURE 4: The population dynamics of the prey-predator system with and without Allee effect. ((a1) and (b1)) Without Allee effect, ((a2) and (b2)) predator with Allee effect, and ((a3) and (b3)) prey with Allee effect. All parameter values are the same as Figure 2.

that the population dynamics of system (1) become more oscillated and complicated when the predator is with Allee effect. However, when the prey is subject to Allee effect, the system becomes more stable.

The corresponding population dynamics (time versus population) of systems (1), (11), and (24) are shown in Figure 4. The paraments are the same as Figure 3. We can clearly conclude that the predator with Allee effect retards the time of system to reach the corresponding equilibrium point, while the prey with Allee effect makes it much faster.

However, when the prey is with too large value of Allee effect $\left(u_{2}=18\right.$ in Figure 5(a) and $u_{2}=23$ in Figure 5(b)), there exists the limited cycle in system (24). In order to obtain a more systematic investigation of system (24), we proceeded to construct bifurcation diagrams of the maximum densities of predator and prey versus $u_{2}$ and $r$, respectively. To do this we first integrated the system numerically with $r=3, \alpha=$ 0.04 , and $\beta=0.04$. According to Theorem 1 , the equilibrium point $\left(x_{0}^{*}, y_{0}^{*}\right)$ of system (1) is asymptotically stable. Letting the system (24) approach the attractor for each value of $u_{2}$, we then plotted successive maxima of both species as a function of $u_{2}$. The resulting bifurcation diagram is plotted in Figure 5. It provides evidence for a qualitative change in the steady state to periodic dynamics and finally to chaotic dynamics as $u_{2}$ is increased.

To construct bifurcation diagrams of the maximum value of prey and predator versus $r$, we do almost the same work as done to obtain those of both species versus $u_{2}$ (Figure 6), except successive maximum densities of predator and prey are plotted of a function of $r$ for $\alpha=0.03, \beta=0.1$, and $u_{2}=18$. Then the periodic dynamics when $0.7<r<1.8$ are showed in Figure 7.

\section{Discussion}

For many years, Allee concept was mentioned as a minor topic in most ecology textbooks but never given very much importance. Over the last two decades, however, Allee concepts began to surface from obscurity with emerging interest in conservation biology and biological invasions. Ecologists started to ponder the dynamics of low-density populations and found that Allee effects can create thresholds below which populations decline toward extinction $[5,6]$. It became apparent that Allee effects are prevalent in low-density populations, arising from a multitude of causes such as matelocation failure, lack of predator satiation, and inability to 


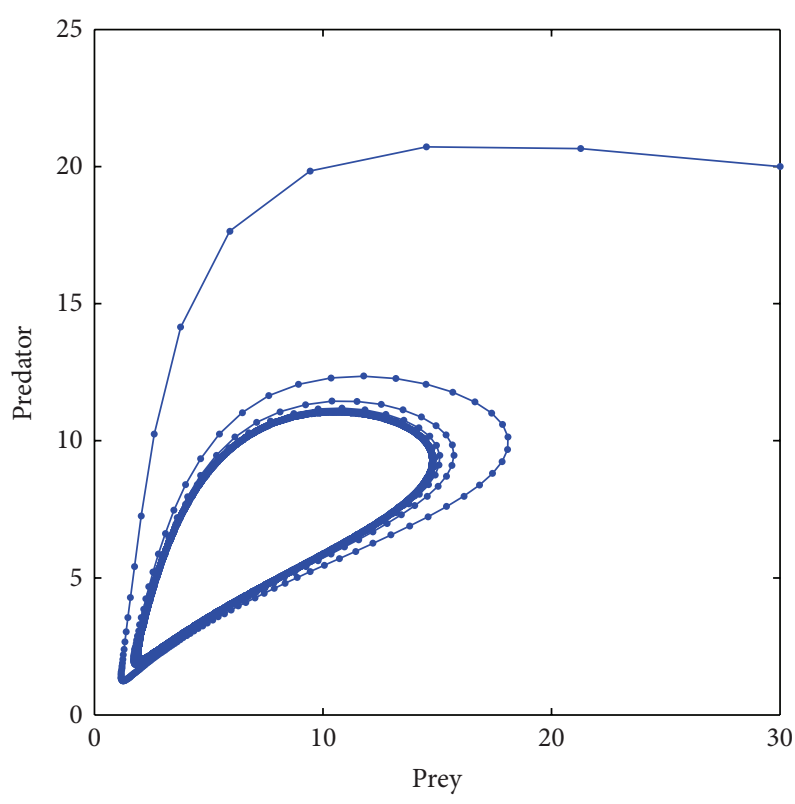

(a)

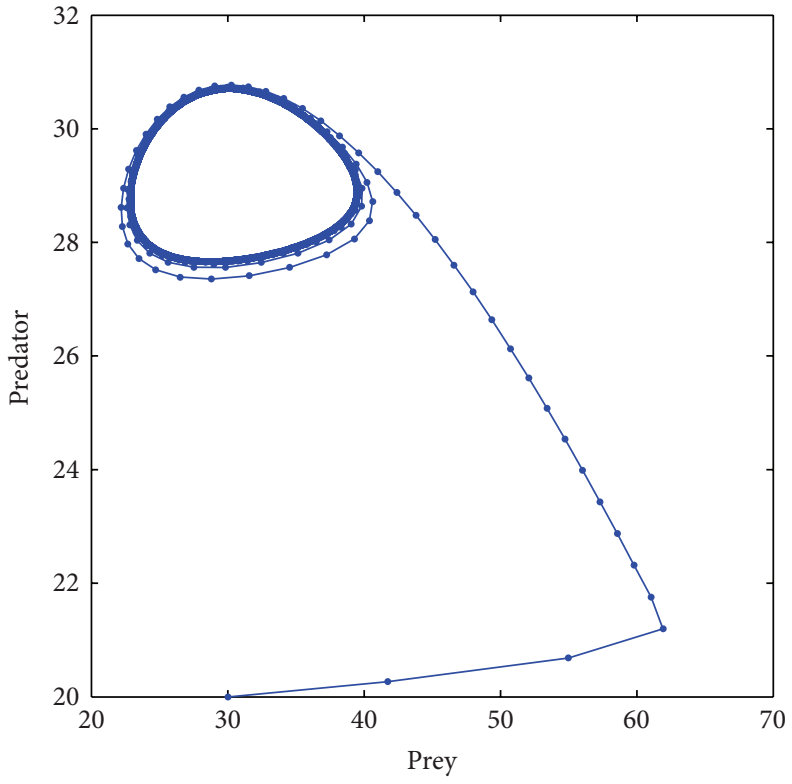

(b)

FIGURE 5: The phase portrait of system (24) by using the initial conditions $x_{0}=30, y_{0}=20$. There is a periodic solution when the prey obeys the Allee effect which gets to a large value. Parameter values: (a) $r=0.7, \alpha=0.03, \beta=0.1, K=100$, and $u_{2}=18$; (b) $r=3, \alpha=0.04$, $\beta=0.04, K=100$, and $u_{2}=23$.

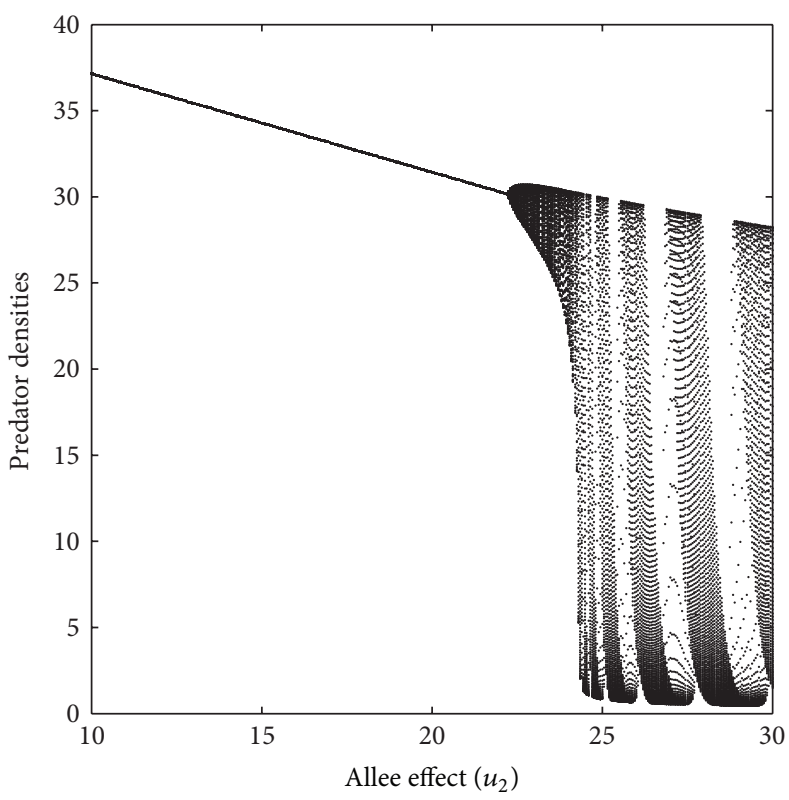

(a)

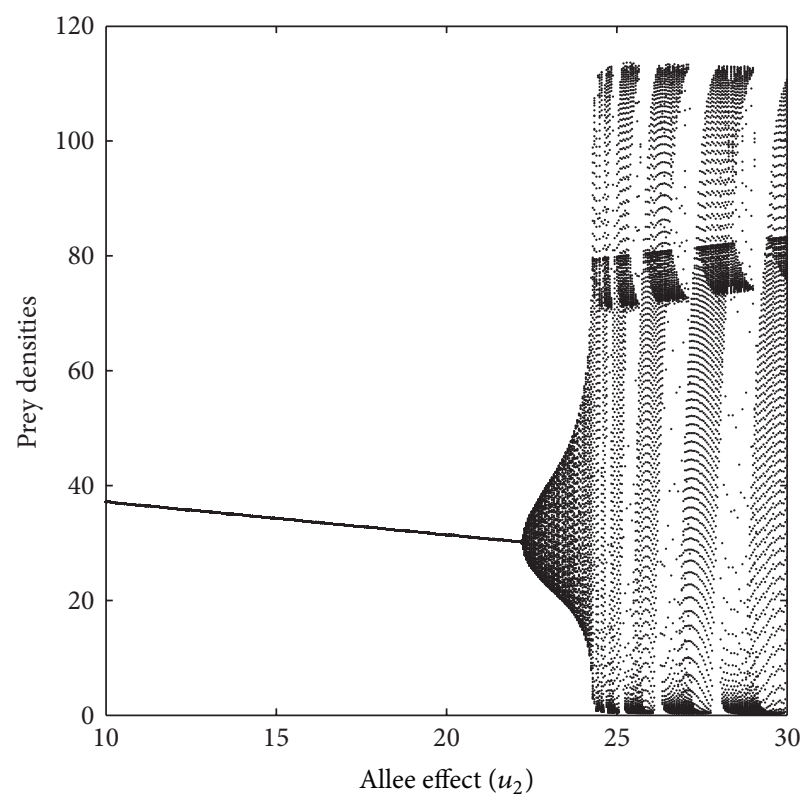

(b)

Figure 6: Bifurcation diagrams of predator and prey densities in model (24) with the initial conditions $x_{0}=30, y_{0}=20$ and the parameter values $r=3, \alpha=0.04, \beta=0.04$, and $K=100$.

engage in group feeding. We now know that Allee effects are critical to understanding the dynamics and persistence of both endangered and invading populations [5, 26, 27].

Previous studies demonstrated that Allee effects play an important role in the stability analysis of equilibrium points of a population dynamics model. An Allee effect may have a stabilizing or a destabilizing effect on population dynamics $[5,7-12,14]$. Even if the system is stable at an equilibrium point, the system subject to an Allee effect may reach its stable state in much longer time. 


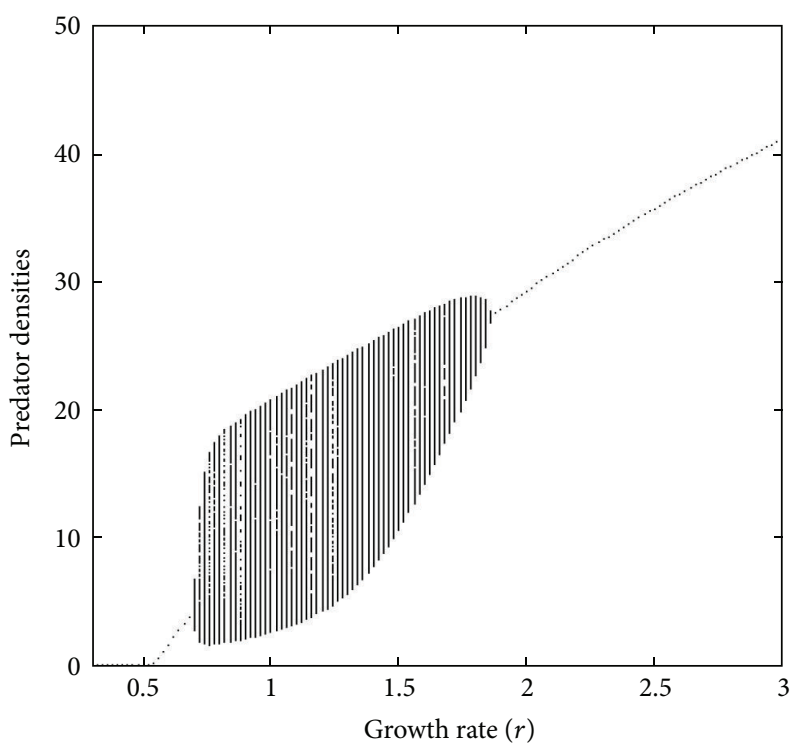

(a)

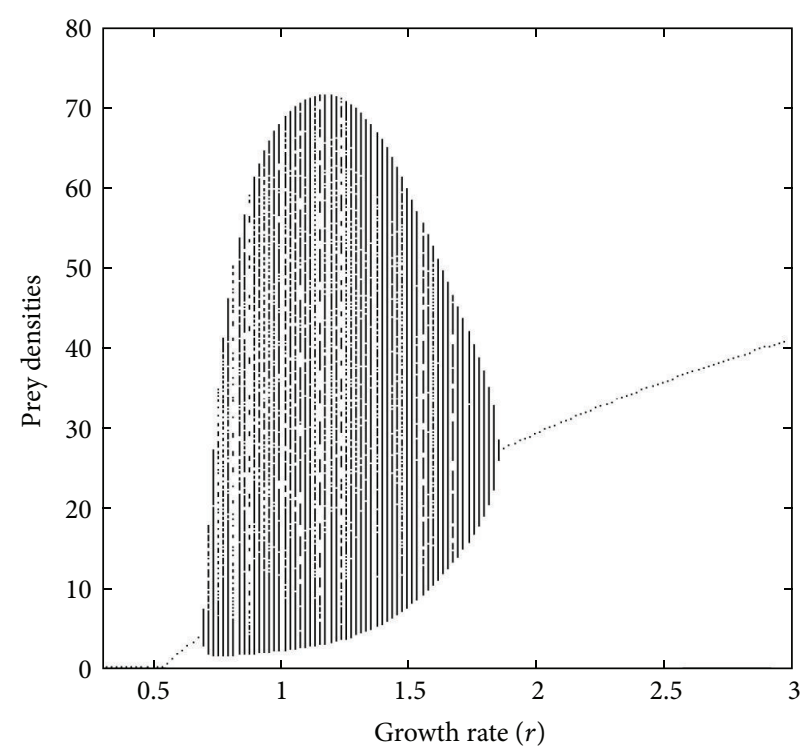

(b)

FIgURE 7: Bifurcation diagrams of predator and prey densities in model (24) with the initial conditions $x_{0}=30, y_{0}=20$ and the parameter values $\alpha=0.03, \beta=0.1, K=100$, and $u_{2}=18$.

This paper focused on the asymmetrical influence of Allee effect on two interacting species, each with nonoverlapping generations. By combining mathematical analysis and numerical simulation, we attempt to clarify the overall characteristics of such systems with and without Allee effect. When the predator obeys Allee effect, the predator-prey system will take longer time to approach the steady state and become more complicated, which is in agreement with that of Zhou et al. [14]. However, when the prey population is with Allee effect, the system may reach the equilibrium point much faster. Here, an increase in stability refers to the case that the system with Allee effect approximates to the equilibrium point much faster than that without it. Clearly, in our system, Allee effects not only stabilize but also destabilize the population dynamics, which is in disagreement with previous work $[10,14,18-21]$. The impact of the Allee effect on the stability of population models shows different dynamics when the corresponding model is difference. We study the predator and prey subject to Allee effect in the same system, respectively, and conclude that the Allee effect has a stabilizing or a destabilizing effect also depending on which species of the interacting system obeys it.

The asymmetrical Allee effects have an important influence on interacting species. Compared the dynamics when the Allee effect on predator with that on prey, we obtain successive new conclusions as follow.

(1) When the predator is with Allee effect, the density of prey species increases and predator decreases in the final equilibrium. The system reaches the stable state in much longer time and the dynamics of it become oscillated and complicated.

(2) When the prey is with Allee effect, both species finally decrease their population densities. The system approaches the stable state much faster. However, a large value of Allee effect on prey results in periodic dynamics which is in disagreement with the results obtained by previous researchers $[5,11,14]$.

In this paper, we investigated the Allee effect which occurs on the predator and prey population, respectively. However, it may be a very complicated structure when both populations are subject to an Allee effect in our system. Thus, in the future studies, it would be very interesting to improve such structures.

\section{Acknowledgments}

The project is supported by the NSF of China (nos. 11226257 and 31170430) and the Fundamental Research Funds for the Central Universities (no. zyz2012074).

\section{References}

[1] F. Courchamp, T. Clutton-Brock, and B. Grenfell, "Inverse density dependence and the Allee effect," Trends in Ecology and Evolution, vol. 14, no. 10, pp. 405-410, 1999.

[2] P. A. Stephens and W. J. Sutherland, "Consequences of the Allee effect for behaviour, ecology and conservation," Trends in Ecology and Evolution, vol. 14, no. 10, pp. 401-405, 1999.

[3] A. P. Møller and S. Legendre, "Allee effect, sexual selection and demographic stochasticity," Oikos, vol. 92, no. 1, pp. 27-34, 2001.

[4] D. S. Boukal and L. Berec, "Single-species models of the Allee effect: extinction boundaries, sex ratios and mate encounters," Journal of Theoretical Biology, vol. 218, no. 3, pp. 375-394, 2002.

[5] B. Dennis, "Allee effects: population growth, critical density, and the chance of extinction," Natural Resource Modeling, vol. 3, no. 4, pp. 481-538, 1989. 
[6] R. Lande, "Extinction thresholds in demographic models of territorial populations.," American Naturalist, vol. 130, no. 4, pp. 624-635, 1987.

[7] M. Gyllenberg, J. Hemminki, and T. Tammaru, "Allee effects can both conserve and create spatial heterogeneity in population densities," Theoretical Population Biology, vol. 56, no. 3, pp. 231242, 1999.

[8] I. Scheuring, "Allee effect increases the dynamical stability of populations," Journal of Theoretical Biology, vol. 199, no. 4, pp. 407-414, 1999.

[9] M. S. Fowler and G. D. Ruxton, "Population dynamic consequences of Allee effects," Journal of Theoretical Biology, vol. 215, no. 1, pp. 39-46, 2002.

[10] C. Çelik, H. Merdan, O. Duman, and Ö. Akın, "Allee effects on population dynamics with delay," Chaos, Solitons \& Fractals, vol. 37, no. 1, pp. 65-74, 2008.

[11] M. A. McCarthy, "The Allee effect, finding mates and theoretical models," Ecological Modelling, vol. 103, no. 1, pp. 99-102, 1997.

[12] P. Amarasekare, "Allee effects in metapopulation dynamics," American Naturalist, vol. 152, no. 2, pp. 298-302, 1998.

[13] C. E. Brassil, "Mean time to extinction of a metapopulation with an Allee effect," Ecological Modelling, vol. 143, no. 1-2, pp. 9-16, 2001.

[14] S. R. Zhou, Y. F. Liu, and G. Wang, "The stability of predatorprey systems subject to the Allee effects," Theoretical Population Biology, vol. 67, no. 1, pp. 23-31, 2005.

[15] G. Wang, X. G. Liang, and F. Z. Wang, "The competitive dynamics of populations subject to an Allee effect," Ecological Modelling, vol. 124, no. 2-3, pp. 183-192, 1999.

[16] F. Courchamp, B. T. Grenfell, and T. H. Clutton-Brock, "Impact of natural enemies on obligately cooperative breeders," Oikos, vol. 91, no. 2, pp. 311-322, 2000.

[17] A. M. de Roos and L. Persson, "Size-dependent life-history traits promote catastrophic collapses of top predators," Proceedings of the National Academy of Sciences of the United States of America, vol. 99, no. 20, pp. 12907-12912, 2002.

[18] A. M. de Roos, L. Persson, and H. R. Thieme, "Emergent Allee effects in top predators feeding on structured prey populations," Proceedings of the Royal Society B, vol. 270, no. 1515, pp. 611-618, 2003.

[19] T. van Kooten, A. M. de Roos, and L. Persson, "Bistability and an Allee effect as emergent consequences of stage-specific predation," Journal of Theoretical Biology, vol. 237, no. 1, pp. 6774, 2005.

[20] C. Çelik and O. Duman, "Allee effect in a discrete-time predatorprey system," Chaos, Solitons \& Fractals, vol. 40, no. 4, pp. 19561962, 2009.

[21] D. Hadjiavgousti and S. Ichtiaroglou, "Allee effect in a preypredator system," Chaos, Solitons \& Fractals, vol. 36, no. 2, pp. 334-342, 2008.

[22] P. H. Leslie, "Some further notes on the use of matrices in population mathematics," Biometrika, vol. 35, pp. 213-245, 1948.

[23] J. D. Murray, Mathematical Biology, vol. 19 of Biomathematics, Springer, Berlin, Germany, 2nd edition, 1993.

[24] W. C. Allee, Animal Aggregations, a Study in General Sociology, University of Chicago Press, Chicago, Ill, USA, 1931.

[25] K. Reinhardt and G. Köhler, "Conservation of the red-winged grasshopper, Oedipoda germanica (Latr.): the influence of reproductive behaviour," Biological Conservation, vol. 107, no. 2, pp. 221-228, 2002.
[26] C. M. Taylor and A. Hastings, "Allee effects in biological invasions," Ecology Letters, vol. 8, pp. 895-908, 2005.

[27] L. Berec, E. Angulo, and F. Courchamp, "Multiple Allee effects and population management," Trends in Ecology and Evolution, vol. 22, no. 4, pp. 185-191, 2007. 


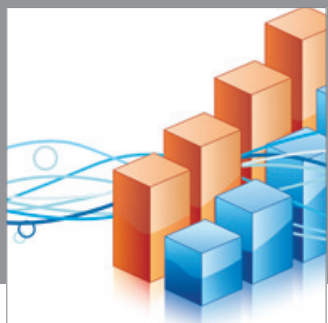

Advances in

Operations Research

mansans

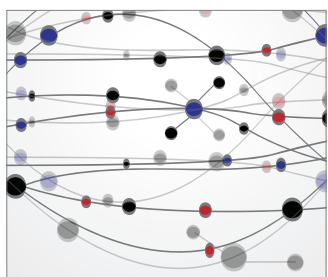

The Scientific World Journal
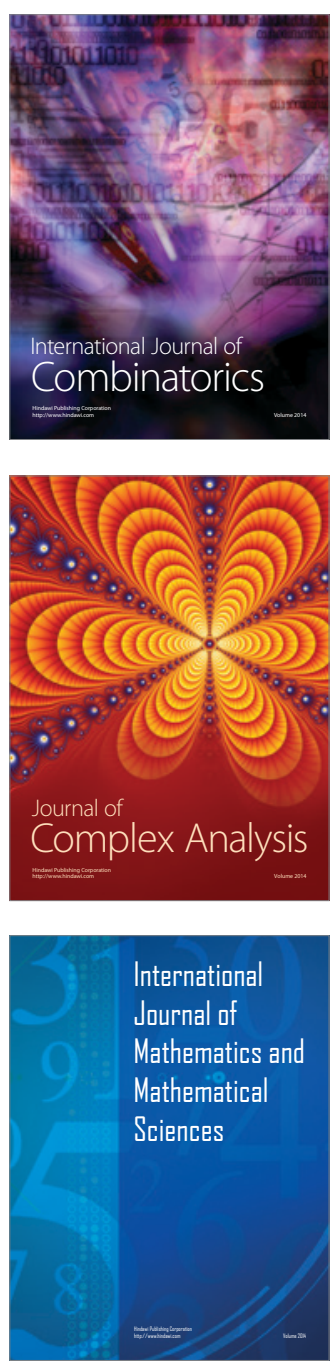
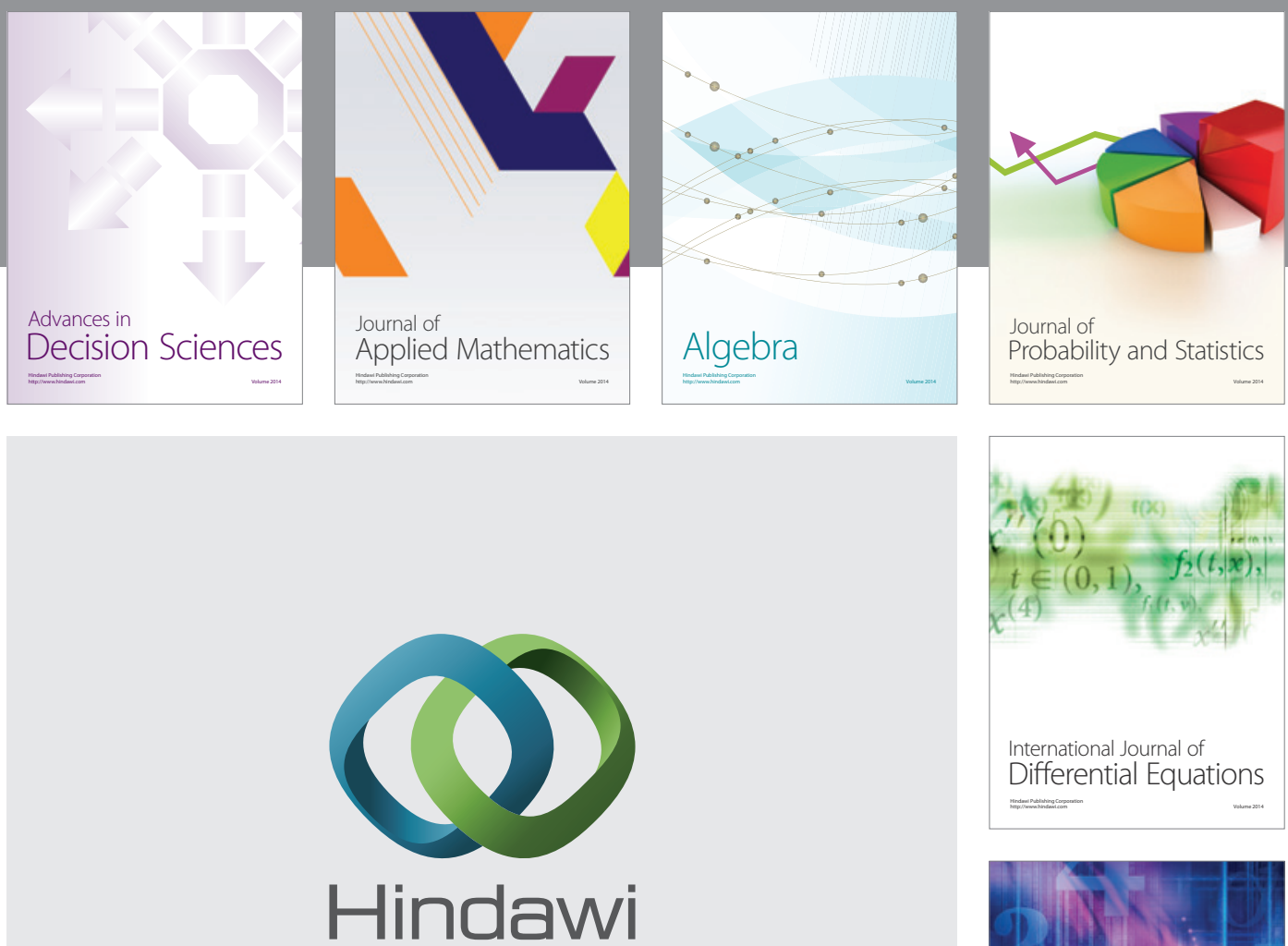

Submit your manuscripts at http://www.hindawi.com
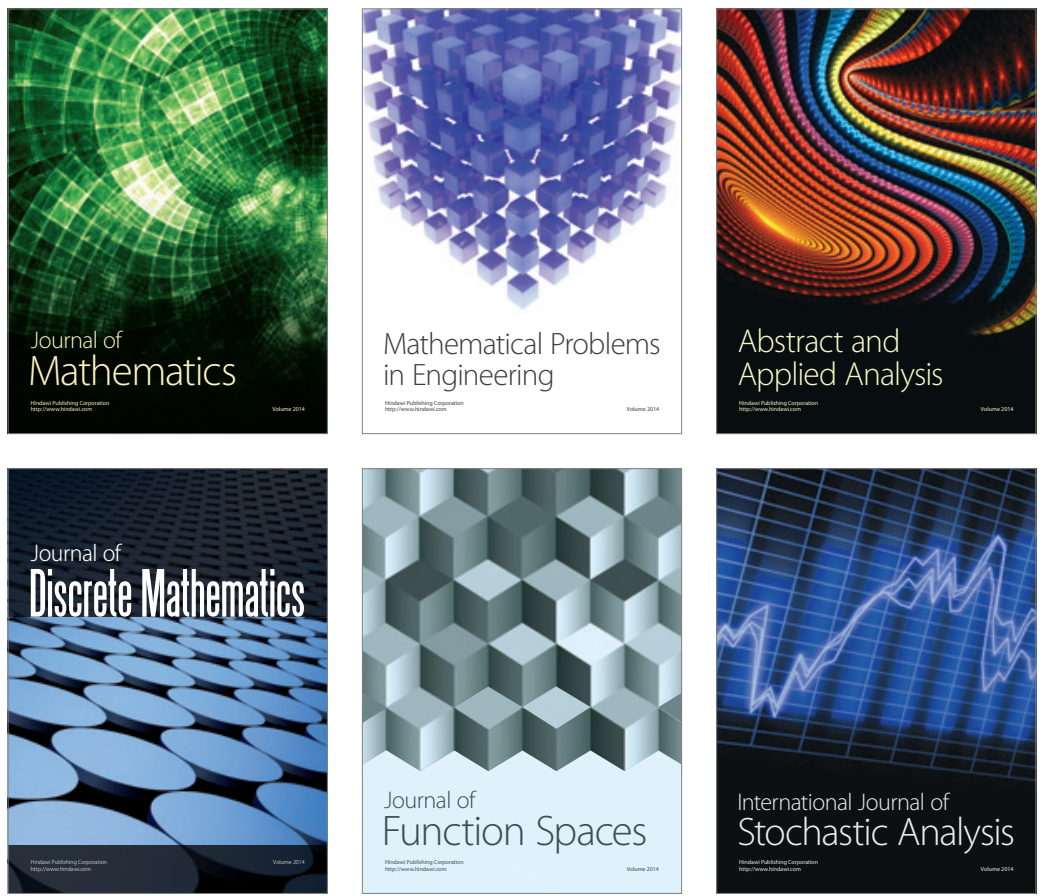

Journal of

Function Spaces

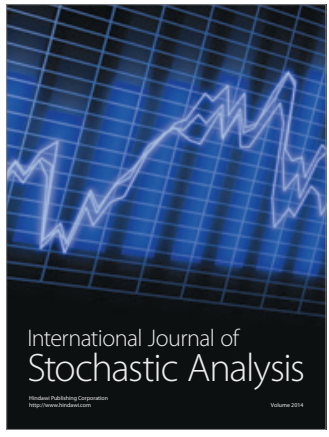

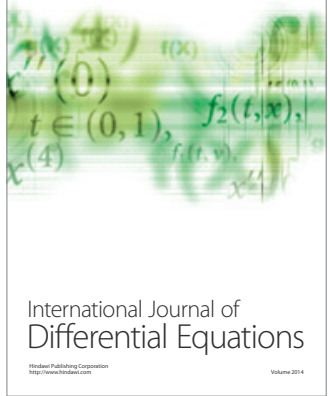
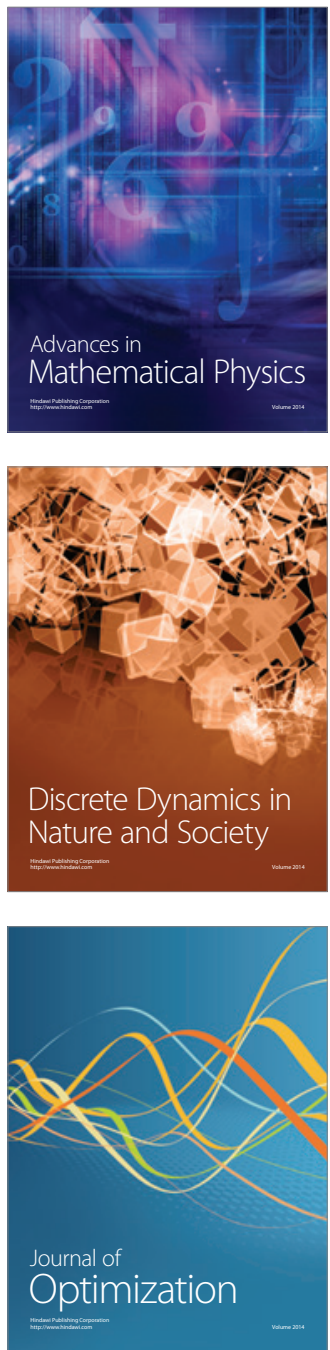